\title{
DETERMINAÇÃO DE HEPARINA FRACIONADA EM PREPARAÇÕES FARMACÊUTICAS UTILIZANDO TURBIDIMETRIA
}

Samantha Sant'Anna Marotta-Oliveira* e Juliana Maldonado Marchetti

Faculdade de Ciências Farmacêuticas de Ribeirão Preto, Universidade de São Paulo, Av. do Café, s/n, 14040-903 Ribeirão Preto $-\mathrm{SP}$, Brasil

Recebido em 17/7/10; aceito em 5/12/10; publicado na web em 29/3/11

\begin{abstract}
DETERMINATION OF FRACTIONATED HEPARIN IN PHARMACEUTICAL DOSAGE FORMS USING TURBIDIMETRY. A turbidimetric method has been used for quantification of fractionated heparin (FH) in pharmaceutical dosage. The UV detection at two wavelengths (290 and $500 \mathrm{~nm}$ ) showed a significant increase in sensitivity of the method, specificity, and linearity to range 5.0$50.0 \mu \mathrm{g} \mathrm{mL}-1$ and 50.0-200.0 $\mu \mathrm{g} \mathrm{mL}^{-1}$, respectively $(\mathrm{r}<0.99)$. At both wavelengths, the method was precise (inter-assay $\mathrm{CV}<5.0 \%$, and intra-assay $\mathrm{CV}<3.0 \%$ ), accurate (maximum deviation of $\pm 12 \%$ ), and robust to the parameters evaluated. Turbidimetry proved to be easy, inexpensive and relatively fast. The results obtained attest to the reliability of the method.
\end{abstract}

Keywords: fractionated heparin; quantitative analysis; turbidimetry.

\section{INTRODUÇÃO}

A heparina é o anticoagulante mais utilizado mundialmente há mais de 50 anos no tratamento e profilaxia da trombose venosa profunda (TVP) e de sua principal complicação, a embolia pulmonar. Constitui o segundo fármaco natural mais utilizado no mundo, superado apenas pela insulina. ${ }^{1}$ Trata-se de uma glicosaminoglicana composta de cadeias de resíduos alternados de D-glicosaminoglicana e um ácido urônico. Sua molécula é sulfatada, ácida e carregada negativamente..$^{2-4}$

As heparinas fracionadas (HFs) são obtidas a partir da heparina extraída da mucosa intestinal de suínos por degradação química ou enzimática. ${ }^{5-7}$ Dentre as HFs disponíveis, a enoxaparina sódica (Figura 1) é a mais estudada e tem sido veiculada em muitas formas farmacêuticas de uso parenteral. Algumas estão disponíveis no mercado como Enoxil ${ }^{\circledR}$ (Prodotti), Dripanina ${ }^{\circledR}$ (Ariston) e Clexane ${ }^{\circledR}$ (Sanofi). Nos últimos anos é crescente o número de pesquisas objetivando o desenvolvimento de novos sistemas de liberação para otimização da terapia. Assim, o desenvolvimento de métodos para o doseamento da HF é bastante relevante.

Diversos métodos analíticos têm sido desenvolvidos para avaliar e quantificar as glicosaminoglicanas incluindo as heparinas. Entretanto, os estudos são dirigidos para a determinação das várias sequências sacarídicas que compõem a molécula e utilizam técnicas como ressonância magnética nuclear, espectrometria de massas e sistemas eletroforéticos. ${ }^{8-13}$ Estes métodos são trabalhosos e se tornam inviáveis para a quantificação da heparina em formas farmacêuticas, pois envolvem processos de derivatização, incluindo a utilização de enzimas e a cromatografia de exclusão para a separação das diferentes frações sacarídicas. A United States Pharmacopeial Convention publicou em 2008 uma monografia atualizada para a HF, porém não descreve metodologia menos laboriosa. Outras formas encontradas para a análise quantitativa da HF são os métodos nefelométrico, descrito por Ardry, ${ }^{14} \mathrm{e}$ turbidimétrico utilizado em trabalhos mais recentes. ${ }^{15-17}$

A turbidimetria é uma técnica relacionada à colorimetria, capaz de

*e-mail: samantha@fcfrp.usp.br

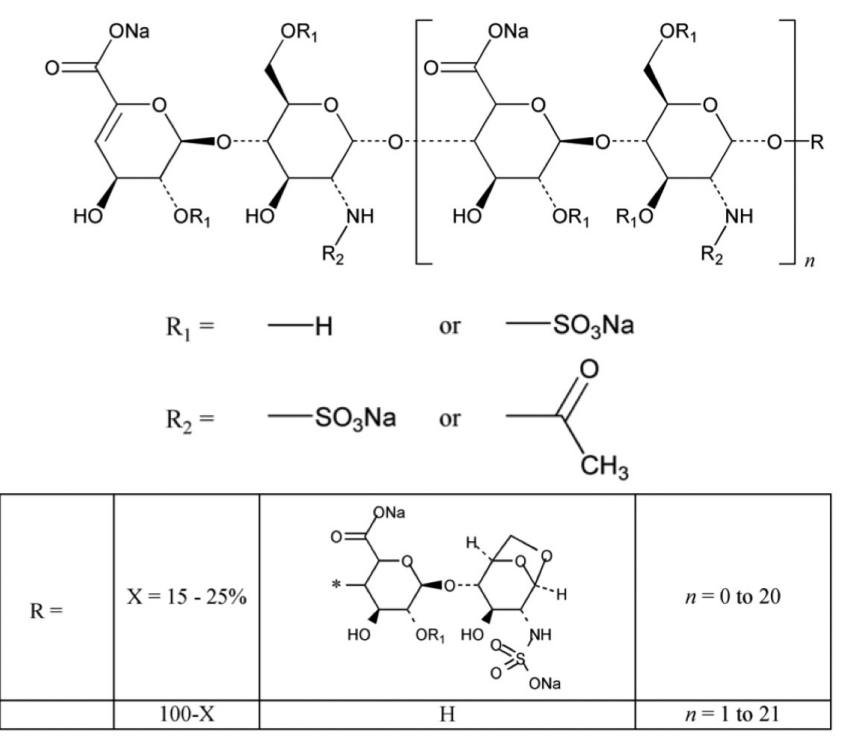

Figura 1. Estrutura química da heparina fracionada (enoxaparina sódica)

medir a turbidez de uma amostra, e possui aplicação restrita à quantificação de substâncias que podem ser precipitadas, utilizando reagentes apropriados, em condições que assegurem a formação de uma suspensão suficientemente estável para se obter reprodutibilidade. ${ }^{18}$

Análises turbidimétricas têm sido utilizadas como alternativa para o doseamento de fármacos em diversas formas farmacêuticas. Farhadi et al. ${ }^{19}$ desenvolveram métodos turbidimétricos para a determinação de fenotiazinas em preparações farmacêuticas utilizando sulfato de dodecil e cloreto de mercúrio como reagentes de precipitação. O emprego de medidas turbidimétricas em sistemas de análise por injeção em fluxo foi utilizado por Suarez et al. ${ }^{20,21}$ e Canaes e Fatibello-Filho ${ }^{22}$ para a determinação de acetilcisteína, cloridrato de fluoxetina e metilbrometo de homatropina, respectivamente, usando nitrato de prata para a precipitação do fármaco. Costa-Neto et al. ${ }^{23}$ desenvolveram um método em fluxo empregando ácido silicotun- 
gstico para a determinação turbidimétrica de tiamina em amostras comerciais. Silveira e Tarley ${ }^{24}$ utilizaram a turbidimetria para a determinação de amitriptilina baseando-se na formação do par iônico AMT-lauril sulfato de sódio usando sistemas em fluxo. Marcolino-Jr. et al. ${ }^{25}$ utilizaram a turbidimetria para a determinação de dipirona em formulações farmacêuticas.

O presente estudo teve como objetivo o desenvolvimento e validação de um método analítico para a quantificação da HF intacta em preparações farmacêuticas empregando a turbidimetria.

\section{PARTE EXPERIMENTAL}

\section{Reagentes e soluções}

A HF utilizada foi a enoxaparina sódica (lote: 034/07), cedida gentilmente pela Kin Master (Passo Fundo, Brasil) e empregada conforme adquirida. Os demais reagentes empregados foram: acetato de sódio (Mallinckrodt LabGuard), cloreto de cetilpiridínio (lote: 42953), cloreto de potássio (lote: 105703), cloreto de sódio (lote: 112987), fosfato de potássio monobásico anidro (lote: 105427) e fosfato de sódio dibásico hepta-hidratado (lote: 93438), grau P.A. (Synth), e ácido acético (lote: 044058), grau cromatográfico (Vetec). A água foi obtida pelo Sistema Milli-Q Plus - Millipore ${ }^{\circledR} /$ Millipore Corporation (EUA).

\section{Métodos}

\section{Solução padrão}

As soluções padrão foram preparadas rigorosamente no mesmo meio de análise do fármaco, a partir de diluições de uma solução estoque da HF (1,0 $\left.\mathrm{mg} \mathrm{mL}^{-1}\right)$ em água Milli-Q.

\section{Desenvolvimento do método analítico}

As determinações turbidimétricas foram realizadas adicionandose em um tubo de vidro $0,5 \mathrm{~mL}$ da amostra contendo a $\mathrm{HF}, 0,5 \mathrm{~mL}$ de solução tampão acetato $1 \mathrm{~mol} \mathrm{~L}^{-1} \mathrm{pH} 4,4$ e $2 \mathrm{~mL}$ de solução de cloreto de cetilpiridínio $0,1 \%(\mathrm{~m} / \mathrm{v})$ e $\mathrm{NaCl} 0,94 \%(\mathrm{~m} / \mathrm{v})$, em triplicata. O branco foi preparado da mesma forma utilizando $0,5 \mathrm{~mL}$ do veículo excetuando a adição do fármaco. Os tubos foram agitados e incubados a $37{ }^{\circ} \mathrm{C}\left( \pm 0,5^{\circ} \mathrm{C}\right)$ por $1 \mathrm{~h}$ em banho termostatizado FANEM 1100 (Brasil). Todas as análises foram realizadas em espectrofotômetro UV - visível Femto 800XI (Brasil) utilizando células de quartzo com caminho óptico de $1 \mathrm{~cm}$ e comprimentos de onda $(\lambda)$ de leitura de 290 ou $500 \mathrm{~nm}$, dependendo da faixa linear obtida nas curvas analíticas.

O método foi desenvolvido e validado para a determinação da HF em micropartículas de poli-(D,L-láctico-co-glicólico) (PLGA), para cálculo da porcentagem de fármaco encapsulado e para sua quantificação em estudos in vitro de liberação. Os meios utilizados para a construção das curvas analíticas foram: meio contendo a mesma composição das micropartículas (PLGA e álcool polivinílico), excetuando o fármaco, e meio composto de tampão fosfato salino $10^{-3} \mathrm{~mol} \mathrm{~L}^{-1} \mathrm{pH} \mathrm{7,4}$ (cloreto de sódio $1,37 \times 10^{-2} \mathrm{~mol} \mathrm{~L}^{-1}$, cloreto de potássio $2,7 \times 10^{-3} \mathrm{~mol} \mathrm{~L}^{-1}$, fosfato de sódio dibásico hepta-hidratado $8,0 \times 10^{-3} \mathrm{~mol} \mathrm{~L}^{-1}$, fosfato de potássio monobásico anidro $2,0 \times 10^{-3}$ mol L ${ }^{-1}$ ), respectivamente. Ambos filtrados em membrana de celulose regenerada $(0,45 \mu \mathrm{m})$.

\section{Validação do método \\ Os parâmetros avaliados seguiram as especificações da ANVISA ${ }^{26}$ e recomendações do ICH Harmonised Tripartite Guideline. ${ }^{27}$}

\section{Seletividade}

A seletividade foi determinada pela comparação dos resultados obtidos de amostras da HF contaminadas com quantidades apropriadas de adjuvantes utilizados na preparação das micropartículas (PLGA, álcool polivinílico e resíduo de diclorometano) e amostras não contaminadas.

\section{Linearidade}

A partir de diluições da solução estoque da $\mathrm{HF}\left(1,0 \mathrm{mg} \mathrm{mL}^{-1}\right)$ foram preparadas soluções em 20 concentrações diferentes, compreendendo de 1,0 a 1.000,0 $\mu \mathrm{g} \mathrm{mL} \mathrm{m}^{-1}$, com leituras em 290 e $500 \mathrm{~nm}$. Os intervalos lineares foram calculados e as curvas analíticas para cada $\lambda$ foram construídas, relacionando os valores de concentração das soluções no eixo das abscissas com os valores de absorvância obtidos no eixo das ordenadas. A proporcionalidade entre a concentração e a resposta foi verificada mediante a obtenção de uma curva padrão e do cálculo da inclinação da reta, do coeficiente de correlação linear (r) e da equação da reta (coeficiente linear, coeficiente angular, soma residual dos mínimos quadrados). O coeficiente de correlação (r) mínimo aceitável foi de $0,99 .^{26}$

\section{Precisão e exatidão}

A precisão e exatidão foram baseadas no método de padronização externa e avaliadas em dois níveis: repetibilidade e precisão intermediária, e exatidão intra e interensaios, respectivamente. Para determinação da repetibilidade e da exatidão intraensaio foram realizadas 10 leituras independentes, em um mesmo dia nas mesmas condições de análises, em três concentrações diferentes para cada comprimento de onda: 5,0; 25,0 e 50,0 $\mu \mathrm{g} \mathrm{mL}^{-1}$ em $290 \mathrm{~nm}$ e 50,0; 100,0 e 200,0 $\mu \mathrm{g} \mathrm{mL}^{-1}$ em $500 \mathrm{~nm}$. Os estudos de exatidão interensaios e precisão intermediária foram realizados em triplicata, para as mesmas concentrações acima, em 5 dias consecutivos. Os cálculos para avaliar a precisão do método foram realizados utilizando-se os gráficos de calibração. As dispersões em relação aos valores médios foram expressas como coeficiente de variação $(\mathrm{CV})$ obtido pela Equação 1.

$$
C V \%=\frac{s}{\bar{X}} \times 100
$$

onde: $s=$ desvio padrão; $\bar{X}=$ média dos valores obtidos.

Para os cálculos da exatidão (E) foram utilizadas as medidas das concentrações obtidas nos estudos intra e interensaios e a concentração real presente na amostra, segundo a Equação 2.

$$
E=\frac{\text { valor obtido }- \text { valorteórico }}{\text { valor teórico }} \times 100
$$

\section{$\underline{\text { Robustez }}$}

Para a avaliação da robustez do método, os ensaios foram submetidos a variações nas condições analíticas. Os parâmetros avaliados foram variação do $\mathrm{pH}$, do tempo e da temperatura da reação e alteração no meio onde o fármaco foi determinado (água, solução tampão e sobrenadante do preparo de micropartículas inertes).

\section{RESULTADOS E DISCUSSÃO}

A HF é uma macromolécula sulfatada, ácida e carregada negativamente. A maioria dos métodos de análise das heparinas descritos na literatura utilizam processos de derivatização para analisar as diferentes frações de oligossacarídeos que constituem a molécula. Entretanto, este processo não é viável para a determinação do fármaco em formas farmacêuticas, principalmente para estudos do perfil de liberação in vitro. A turbidimetria foi a técnica de escolha para a análise quantitativa da $\mathrm{HF}$, devido ao insucesso por cromatografia 
líquida de alta eficiência (CLAE), incluindo técnicas como cromatografia de par iônico, troca iônica, exclusão utilizando detector por UV-vis e índice de refração.

Turbidimetria é uma expressão da propriedade ótica de uma amostra que faz com que a radiação seja dispersa e absorvida antes de ser transmitida em linha reta através da amostra. ${ }^{28}$ Quanto à instrumentação, o turbidímetro mede a quantidade de radiação que passa através de uma amostra, análogo à espectrofotometria de absorção, e pode ser realizada em colorímetros e espectrofotômetros sem nenhuma modificação. ${ }^{18,28}$ Entretanto, a determinação analítica deve ser empírica, pois diferenças no projeto físico de um instrumento causam diferenças nos valores medidos para a turdidez, ainda que o mesmo material de calibração seja usado para cada instrumento. ${ }^{28} \mathrm{~A}$ exatidão das técnicas turbidimétricas pode ser comprometida devido à dificuldade em assegurar que o tamanho de partículas em uma suspensão, após a precipitação, seja o mesmo durante cada medida como durante a calibração. Os erros que ocorrem durante a medida são geralmente insignificantes em comparação com aqueles devido à falta de reprodutibilidade das propriedades da suspensão por falha na padronização. A concentração dos reagentes, ordem de preparação da amostra, agitação, o tempo posterior à reação e anterior às leituras são fatores que afetam os resultados e devem ser rigorosamente controlados. $\mathrm{O} \mathrm{pH}$, a força iônica, a temperatura e a presença de impurezas são outras variáveis críticas a serem controladas. ${ }^{28,29}$ Assim, foi necessário padronizar todas as etapas e preparar a curva analítica e o branco da amostra sempre nas mesmas condições das amostras para todas as análises, a fim de minimizar interferências.

Neste estudo, a análise turbidimética baseia-se na reação de precipitação de estequiometria (1:1). ${ }^{30} \mathrm{Em} \mathrm{pH}$ ácido, os grupos sulfatados da HF (Figura 1), carregados negativamente, reagem com os grupos de amônio quaternário das moléculas de cloreto de cetilpiridínio (Figura 2a), carregados positivamente, formando pares iônicos. As cadeias carbônicas longas, presentes nas moléculas do cloreto de cetilpiridínio que se ligam à $\mathrm{HF}$, geram um composto estruturalmente complexo (Figura 2b), que precipita, deixando o meio reacional turvo. A turbidez da amostra é diretamente proporcional à concentração de HF no meio.

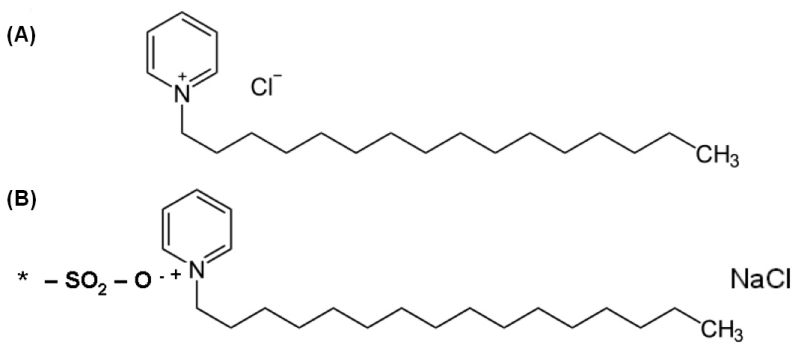

Figura 2. Estrutura química do cloreto de cetilpiridínio (a) e da ligação dos grupos sulfatados* da HF ao cloreto de cetilpiridínio que formam o composto insolúvel ( $b$ )

As amostras foram analisadas em vários comprimentos de onda $(\lambda)$ a fim de definir o intervalo linear. Os primeiros testes foram realizados utilizando $\lambda=500 \mathrm{~nm}$ e apresentou linearidade na faixa de 50,0 a $200,0 \mu \mathrm{L} \mathrm{mL}^{-1}(r=0,9962)$. Esta faixa linear foi ideal para a determinação da eficiência de encapsulação da HF nas micropartículas de PLGA, porém não alcançou sensibilidade necessária para a os ensaios de liberação in vitro do fármaco microencapsulado. De acordo com Charlot ${ }^{18}$ e Morais et al..$^{28}$ a sensibilidade da técnica turbidimétrica pode ser aumentada modificando-se o $\lambda$. Assim, as análises também foram realizadas em um $\lambda$ mais baixo $(290 \mathrm{~nm})$, o qual apresentou linearidade na faixa de 5,0 a 50,0 $\mu \mathrm{L} \mathrm{mL}^{-1}(r=0.9963)$.
Os resultados preliminares das curvas analíticas das soluções padrão apresentaram boa correlação nas concentrações analisadas, em conformidade com as exigências da ANVISA. ${ }^{26} \mathrm{O}$ método turbidimétrico foi então validado para os $\lambda 290$ e $500 \mathrm{~nm}$.

\section{Validação do método}

\section{Seletividade}

Segundo a ANVISA, ${ }^{26}$ para a análise quantitativa de fármacos em formas farmacêuticas, a seletividade pode ser determinada pela comparação entre os resultados obtidos de amostras não contaminadas e os obtidos das amostras contendo o fármaco, contaminadas com quantidades apropriadas de adjuvantes, para demonstrar que o resultado do teste não é afetado por esses materiais. Os resultados obtidos para as soluções de HF preparadas em água Milli Q, em tampão fosfato salino $10 \mathrm{mM}$ e no meio contendo os adjuvantes das micropartículas de PLGA demonstraram não serem afetados pelas matérias-primas utilizadas.

\section{Linearidade}

A linearidade do método foi determinada através de curvas analíticas (n=3) utilizando concentrações superiores àquelas empregadas para a construção do gráfico de calibração. Após o ajuste dos dados por regressão linear utilizando o método dos mínimos quadrados, as equações analíticas foram determinadas e as respostas obtidas mostraram ser diretamente proporcionais à concentração da amostra para os intervalos compreendidos entre 5,0 a 50,0 $\mu \mathrm{g} \mathrm{mL}^{-1}$ [absorvância $=$ $0,0050 \times$ concentração $\left.\left(\mu \mathrm{g} \mathrm{mL}^{-1}\right)+0,0003\right]$, para leitura em $290 \mathrm{~nm}(\mathrm{r}=$ 0,9963) (Figura 3a), e entre 50,0 a 200,0 $\mu \mathrm{g} \mathrm{mL}^{-1}$ [absorvância $=0,0029$ x concentração $\left(\mu \mathrm{g} \mathrm{mL}^{-1}\right)+0,0015$, para leitura em $500 \mathrm{~nm}(\mathrm{r}=0,9975)$ (Figura 4a). A construção da curva analítica relativa (Figuras 3a e 4b), relacionando os valores de absorvância divididos pelas respectivas concentrações no eixo y e as concentrações correspondentes em escala logarítmica no eixo $\mathrm{x},{ }^{31}$ demonstrou a linearidade para as mesmas faixas de concentração considerando os pontos entre 95 ou $105 \%$ da resposta relativa média. A construção da curva com a concentração em escala logarítmica permitiu melhor visualização da faixa linear.
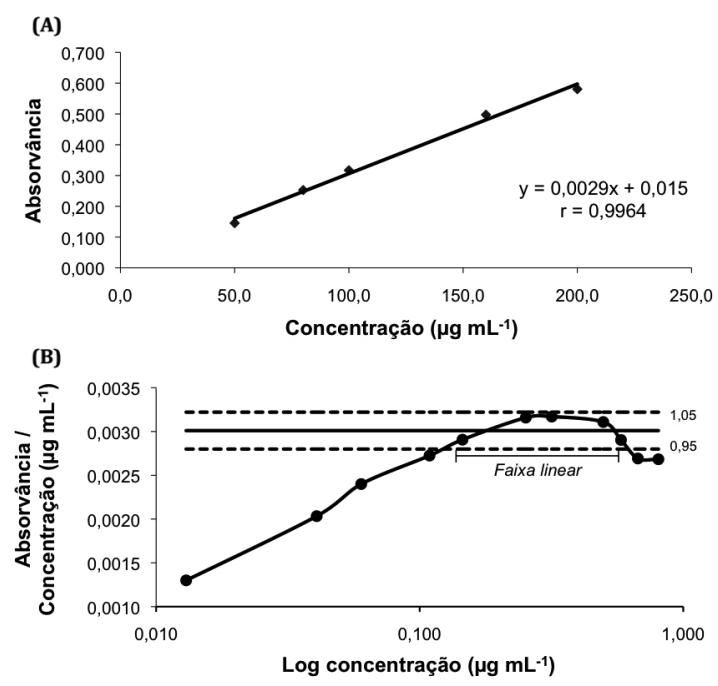

Figura 3. Determinação da linearidade: (A) curva analítica; (B) absorvâncial concentração vs. concentração em escala logarítmica (leitura em $500 \mathrm{~nm}$ )

\section{Precisão e exatidão}

Os resultados obtidos nos estudos de repetibilidade apresentaram CV máximo de 2,95 e 0,84\% utilizando 290 e $500 \mathrm{~nm}$, respectivamente. Já para a precisão intermediária o CV máximo foi de 4,80 

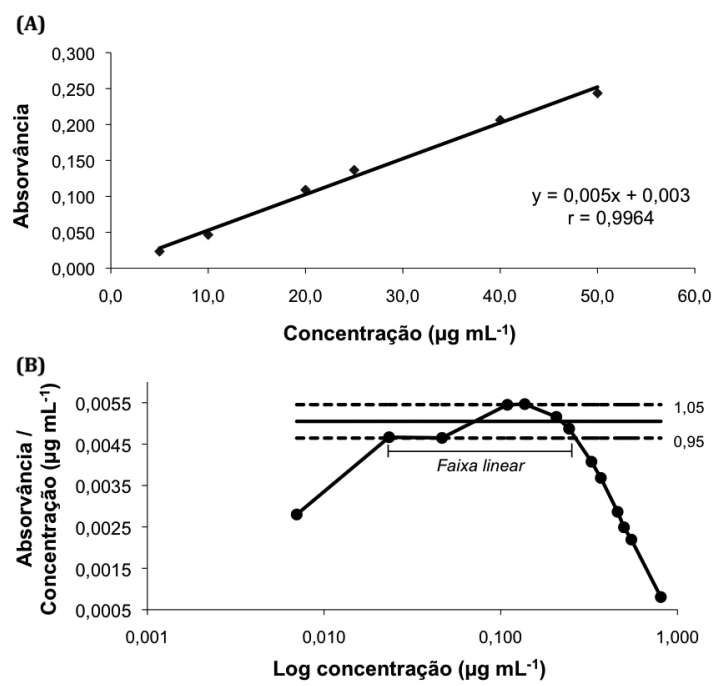

Figura 4. Determinação da linearidade: (A) curva analítica; (B) gráfico da absorvância/concentração vs. concentração em escala logarítmica (leitura em $290 \mathrm{~nm}$ )

e $0,91 \%$, respectivamente (Tabela 1 ). Os resultados obtidos apresentaram valores abaixo de $5 \%$, indicando que o método é preciso.

Tabela 1. Precisão do método turbidimétrico utilizado na análise da heparina fracionada

\begin{tabular}{lcccccc}
\hline \multicolumn{3}{c}{ Parâmetros } & \multicolumn{3}{c}{$500 \mathrm{~nm}$} \\
\hline Repetibilidade & \multicolumn{3}{c}{$290 \mathrm{~nm}$} & 50,0 & 100,0 & 200,0 \\
\hline Conc. teórica $\left(\mu \mathrm{g} \mathrm{mL}^{-1}\right)$ & 10,0 & 25,0 & 50,0 & 10 & 10 & 10 \\
$\mathrm{n}$ & 10 & 10 & 10 & 10 & 0,43 & 0,59 \\
$\mathrm{CV}(\%)$ & 2,95 & 1,00 & 0,76 & 0,84 & 0,43 \\
\hline Precisão intermediária & \multicolumn{3}{c}{$290 \mathrm{~nm}$} & & $500 \mathrm{~nm}$ & \\
\hline Conc. teórica $\left(\mu \mathrm{g} \mathrm{mL}^{-1}\right)$ & 10,0 & 25,0 & 50,0 & 50,0 & 100,0 & 200,0 \\
$\mathrm{n}$ & 3 & 3 & 3 & 3 & 3 & 3 \\
$\mathrm{CV}(\%)$ & 4,80 & 2,63 & 2,56 & 0,91 & 0,34 & 0,89 \\
\hline $\mathrm{C}$ & & & $\mathrm{CV}:$ & & &
\end{tabular}

Conc.: concentração; n: número de replicatas; $\mathrm{CV}$ : coeficiente de variação.

A avaliação da exatidão intra e interensaios (Tabela 2) revelou que os desvios do valor nominal foram menores que $\pm 15 \%$, valores preconizados nos estudos de Causon ${ }^{32}$ e Shah et al., ${ }^{33}$ demonstrando uma boa exatidão do método.

Tabela 2. Exatidão do método turbidimétrico utilizado na análise da heparina fracionada

\begin{tabular}{lcccccc}
\hline \multicolumn{7}{c}{ Parâmetros } \\
\hline Exatidão intra-ensaios & \multicolumn{3}{c}{$290 \mathrm{~nm}$} & \multicolumn{3}{c}{$500 \mathrm{~nm}$} \\
\hline Conc. teórica $\left(\mu \mathrm{g} \mathrm{mL}^{-1}\right)$ & 10,0 & 25,0 & 50,0 & 50,0 & 100,0 & 200,0 \\
Conc. obtida $\left(\mu \mathrm{g} \mathrm{mL}^{-1}\right)$ & 9,8 & 25,1 & 50,1 & 48,9 & 113,8 & 197,9 \\
$\mathrm{n}$ & 10 & 10 & 10 & 10 & 10 & 10 \\
$\mathrm{E}(\%)$ & $-2,08$ & 0,55 & 0,13 & $-2,20$ & 11,79 & $-1,05$ \\
\hline Exatidão inter-ensaios & $290 \mathrm{~nm}$ & \multicolumn{5}{c}{$500 \mathrm{~nm}$} \\
\hline Conc. teórica $\left(\mu \mathrm{g} \mathrm{mL}^{-1}\right)$ & 10,0 & 25,0 & 50,0 & 50,0 & 100,0 & 200,0 \\
Conc. obtida $\left(\mu \mathrm{gL}^{-1}\right)$ & 9,2 & 26,5 & 47,4 & 47,9 & 112,1 & 196,2 \\
$\mathrm{n}$ & 3 & 3 & 3 & 3 & 3 & 3 \\
E(\%) & $-8,20$ & 6,15 & $-5,29$ & $-4,20$ & 12,00 & $-1,90$ \\
\hline Con.
\end{tabular}

Conc.:concentração; n: número de replicatas; E: exatidão.

\section{Robustez}

A robustez de um método indica a capacidade da técnica desenvolvida em resistir a pequenas variações dos parâmetros analíticos, bem como sua confiabilidade durante o uso. ${ }^{26}$ As variações nos parâmetros de análise estabelecidos (Tabela 3) apresentaram um CV máximo de 5,08\%.

Tabela 3. Avaliação da robustez do método turbidimétrico utilizado para a análise da heparina fracionada

\begin{tabular}{ccc}
\hline & $290 \mathrm{~nm}$ & $500 \mathrm{~nm}$ \\
\hline $\mathrm{pH}$ do tampão de reação & $\mathrm{r}$ & $\mathrm{r}$ \\
\hline 4,4 & 0,9963 & 0,9975 \\
3,4 & 0,9962 & 0,9972 \\
5,4 & 0,9960 & 0,9974 \\
$\mathrm{CV}(\%)$ & 0,02 & 0,02 \\
\hline Temperatura de incubação & $\mathrm{r}$ & $\mathrm{r}$ \\
\hline $37^{\circ} \mathrm{C}$ & 0,9963 & 0,9975 \\
$39^{\circ} \mathrm{C}$ & 0,9963 & 0,9960 \\
$36^{\circ} \mathrm{C}$ & 0,9958 & 0,9976 \\
$\mathrm{CV}(\%)$ & 0,03 & 0,09 \\
\hline Meio de análise da HF & $\mathrm{r}$ & $\mathrm{r}$ \\
\hline Água & 0,9965 & 0,9962 \\
Solução tampão fosfato salino 10 mM pH 7,4 & 0,9963 & 0,9975 \\
Meio contendo adjuvantes das & 0,9919 & 0,9988 \\
micropartículas inertes & & \\
CV(\%) & 0,26 & 0,13 \\
\hline
\end{tabular}

\section{CONCLUSÃO}

O método analítico empregando turbidimetria permitiu uma rápida detecção e quantificação da HF. A detecção por UV em 290 $\mathrm{nm}$ apresentou um aumento significativo na sensibilidade do método comparado aos resultados obtidos utilizando $500 \mathrm{~nm}$. Os resultados também indicaram que este método foi seletivo frente aos componentes da formulação e do meio de dissolução utilizado, preciso (CV\% inferior a 5,0\%), exato ( $\mathrm{E} \%$ máximo de $12,0 \%$ ), linear em ampla faixa de concentração $\left(5,0\right.$ a $50,0 \mu \mathrm{g} \mathrm{mL}^{-1}$ e 50,0 a $200,0 \mu \mathrm{g} \mathrm{mL} \mathrm{m}^{-1}$, para leituras em 290 e $500 \mathrm{~nm}$, respectivamente) e robusto para os parâmetros avaliados. A turbidimetria mostrou-se relevante para a determinação de HF, devido à dificuldade de análise por outras técnicas e à escassez de trabalhos publicados. O método desenvolvido e validado demonstrou ser de fácil execução, baixo custo, relativamente rápido e pouco poluente. Os resultados obtidos no presente trabalho atestam a confiabilidade do método para ser utilizado no doseamento da heparina fracionada na forma farmacêutica analisada.

\section{AGRADECIMENTOS}

Ao suporte financeiro da FAPESP e da CAPES. À Kin Master (Brasil) pelo fornecimento da Enoxaparina sódica. À FCFRP-USP. À Prof ${ }^{\mathrm{a}}$. Dr ${ }^{\mathrm{a}}$. D. C. R. de Oliveira, do Departamento de Física e Química, FCFRP-USP, pelas correções nas estruturas químicas e reações e auxílio na discussão.

\section{REFERÊNCIAS}

1. Melo, E. I.; Pereira, M. S.; Cunha, R. S.; Sá, M. P. L.; Mourão, P. A. S.; Rev. Bras. Cir. Cardiovasc. 2008, 23, 169.

2. Majerus, P. W.; Tollefsen, D. M. Em Goodman \& Gilman - As Bases Farmacológicas da Terapêutica; Gilman, A. G.; Hardman, J. G.; Limbird, L. E., eds.; McGraw-Hill: Rio de Janeiro, $10^{\mathrm{a}}$ ed., 2007, cap. 55. 
3. Hirsh, J.; Raschke, R.; Chest 2004, 126, $188 \mathrm{~S}$.

4. Page, C. P.; Curtis, M. J.; Sutter M. C.; Walker, M. J. A.; Hoffman, B. B.; Farmacologia Integrada, Manole: São Paulo, 1999.

5. Hirsh, J.; Bates, S. M.; Progr. Cardiovasc. Dis. 2000, 42, 235.

6. Lensing, A. W. A; Prandoni, P. P.; Prins, M. H.; Büller, H. R.; The Lancet 1999, 353, 479.

7. Weitz, J. I.; N. Engl. J. Med. 1997, 337, 688.

8. Mourier, P. A. J.; Viskov, C.; Anal. Biochem. 2004, 332, 299.

9. Thanawiroon, C.; Rice, K. G.; Toida, T.; Linhardt, R. J.; J. Biol. Chem. 2004, 279, 2608.

10. Thanawiroon, C., Linhardt, R. J.; J. Chromatogr. 2003, 1014, 215.

11. Volpi, N.; Maccari, F.; J. Chromatogr, B: Anal. Technol. Biomed. Life Sci. 2006, 834, 1 .

12. Camara, J. E.; Satterfield, M. B.; Nelson, B. C.; J. Pharm. Biomed. Anal. 2007, 43, 1706; Keire, D. A.; Trehy, M. L.; Reepmeyer, J. C.; Kolinski, R. E.; Ye, W.; Dunn, J.; Westenberger, B. J.; Buhse, L. F.; J. Pharm. Biomed. Anal. 2010, 51, 921; Mourier, P. A. J.; Guichard, O. Y.; Herman, F.; Viskov, C.; J. Pharm. Biomed. Anal. 2011, 54, 337.

13. Patel, R. P.; Narkowicz, C.; Hutchinson, J. P.; Hilder, E. F.; Jacobson, G. A.; J. Pharm. Biomed. Anal. 2008, 46, 30; Liu, Z.; Xiao, Z.; Masuko, S.; Zhao, W.; Sterner, E.; Bansal, V.; Fareed, J.; Dordick, J.; Zhang, F.; Linhardt, R. J.; Anal. Biochem. 2011, 408, 147.

14. Ardry, M.; Bulletin de l'Ordre des Pharmaciens 1971, 135, 699.

15. Hoffart, V.; Lamprecht, A.; Maincent, P.; Lecompte, T.; Vigneron, C.; Ubrich, N.; J. Controlled Release 2006, 113, 38.

16. Hoffart, V.; Ubrich, N.; Lamprecht, A.; Bachelier, K.; Vigneron, C.; Lecompte, T.; Hoffman, M.; Maincent, P.; Drug Delivery 2003, 10, 1; Scala-Bertola, J.; Rabiskova, M.; Lecompte, T.; Bonneaux F.; Maincent, P.; Int. J. Pharm. Sci. 2009, 374, 1217. Ubrich, N.; Maincent, P. Em Microencapsulation: methods and industrial applications; Benita, S., ed.; $2^{\text {nd }}$ ed., CRC Press: Boca Raton, 2006, chap. 14.

18. Charlot, G.; Colorimetric determination of elements: principles and methods, Elsevier Publishing Company: Amsterdam, 1964, chap. 6.

19. Farhadi, K.; Savojbolaghi, A. K.; Farajzadeh, M.; Maleki, R.; Anal. Lett. 2003, 36, 2183.
20. Suarez, W. T.; Vieira, H. J.; Fatibello-Filho, O.; J. Braz. Chem. Soc. 2007, 18, 1028 .

21. Suarez, W. T.; Sartori, E. R.; Batista, E. F.; Fatibello-Filho, O.; Quim. Nova 2009, 32, 2396.

22. Canaes, L. S.; Fatibello-Filho, O.; Quim. Nova 2006, 29, 1237.

23. Costa-Neto, C. O.; Pereira, A. V.; Aniceto, C.; Fatibello-Filho, O.; Talanta 1999, 48, 659.

24. Silveira, G.; Tarley, C. R. T.; Quim. Nova 2008, 31, 1653.

25. Marcolino-Jr., L. H.; Bonifácio, V. G.; Fatibello-Filho, O.; Teixeira, M. F. S.; Quim. Nova 2005, 28, 783.

26. Brasil, Agência Nacional de Vigilância Sanitária (ANVISA); Resolução $n^{o}$ RE 899, de 29 de maio de 2003.

27. ICH - International Conference on Harmonisation of Technical Requirements for Registration of Pharmaceuticals for Human Use; ICH Harmonised Tripartite Guideline. Validation of analytical procedures: text and methodology Q2(R1), 2005.

28. Willard, H. H.; Merritt Jr., L. L.; Dean, J. A.; Settle Jr., F. A.; Instrumental methods of analysis, Wadsworth: Belmont, $7^{\text {th }}$ ed., 1988, chap. 7; Morais, I. P. A.; Tóth, I. V.; Rangel, A. O. S.; Spectrosc. Lett. 2006, $39,547$.

29. Harris, D. C.; Análise Química Quantitativa, Livros Técnicos e Científicos: Rio de Janeiro, $5^{\mathrm{a}}$ ed., 2001.

30. Demoré, B.; Benoit, E.; Maincent, P.; Hoffman, M.; Bessière, J.; J. Clin. Pharm. Ther. 1998, 23, 381; Javot, L.; Lecompte, T.; Rabiskova, M.; Maincent, P.; J. Controlled Release 2009, 139, 8.

31. Ribani, M.; Bottoli, C.; Collins, C. H.; Jardim, I. C. S. F.; Melo, L.; Quim. Nova 2004, 27, 771 .

32. Causon, R.; J. Chromatogr., B: Anal. Technol. Biomed. Life Sci. 1997, 689, 175.

33. Shah, V. P.; Midha, K. K.; Dighe, S.; McGilveray, J. J.; Skelly, J. P.; Yacobi, A.; Layloff, T.; Viswanathan, C. T.; Cook, C. E.; McDowall, R. D.; Pittman, K. A.; Spector, S.; J. Pharm. Sci. 1992, 81, 309. 